\title{
Comparator and half adder design using complementary resistive switches crossbar
}

\author{
Bosheng Liu ${ }^{1}$, Zhiqiang You ${ }^{1 \mathrm{a})}$, Xiangrao $\mathrm{Li}^{2}$, Jishun Kuang ${ }^{1}$, \\ and Zheng Qin ${ }^{1}$ \\ ${ }^{1}$ College of Information Science \& Engineering, Hunan University, 410082 \\ ${ }^{2}$ School of Software, Central South University, 410075 \\ a)you@hnu.edu.cn
}

\begin{abstract}
This paper designs a one-bit comparator and a one-bit half adder using complementary resistive switches (CRS) crossbar units. At first, the finite state machine (FSM) of the crossbar unit with two CRS cells is presented. Then, we demonstrate that the crossbar unit with a single CRS cell can realize the one-bit comparator in 7 sequential cycles, and the crossbar unit with two CRS cells which are connected via a wired AND (' $\&$ ') can realize the one-bit half adder in 5 sequential cycles. Simulation results show that the one-bit comparator and the one-bit half adder can be designed to provide device area benefits via CRS crossbar units. The designs of one-bit comparator and one-bit half adder project the possibility of applications for complex circuit designs with CRS crossbar units.
\end{abstract}

Keywords: complementary resistive switches (CRS), crossbar, circuit design, finite state machine (FSM), memristor

Classification: Integrated circuits

\section{References}

[1] B. Govoreanu, G. S. Kar, Y.-Y. Chen, V. Paraschiv, S. Kubicek, A. Fantini, I. P. Radu, L. Goux, S. Clima, R. Degraeve, N. Jossart, O. Richard, T. Vandeweyer, K. Seo, P. Hendrickx, G. Pourtois, H. Bender, L. Altimime, D. J. Wouters, J. A. Kittl and M. Jurczak: IEEE International Electron Devices Meeting (2011) 729.

[2] M. Lee, S. Kim, C. Lee, H. Yin, S. Ahn, B. Kang, K. Kim, J. Park, C. Kim, I. Song, S. Kim, G. Stefanovich, J. Lee, S. Chung, Y. Kim and Y. Park: Adv. Funct. Mater. 19 [10] (2009) 1587.

[3] W. Y. Park, G. H. Kim, J. Y. Seok, K. M. Kim, S. J. Song, M. H. Lee and C. S. Hwang: Nanotechnology 21 [19] (2010) 195201.

[4] E. Linn, R. Rosezin, C. Kügeler and R. Waser: Nat. Mater. 9 [5] (2010) 403.

[5] R. Rosezin, E. Linn, L. Nielen, C. Kügeler, R. Bruchhaus and R. Waser: IEEE Electron Device Lett. 32 [2] (2011) 191.

[6] J. Borghetti, G. S. Snider, P. J. Kuekes, J. J. Yang, D. R. Stewart and R. S. Williams: Nature 464 [7290] (2010) 873. 
[8] E. Linn, R. Rosezin, S. Tappertzhofen, U. Böttger and R. Waser: Nanotechnology 23 [30] (2012) 305205.

[9] C. Yakopcic, T. M. Taha, G. Subramanyam, R. E. Pino and S. Rogers: IEEE Electron Device Lett. 32 [10] (2011) 1436.

[10] C. Yakopcic, T. M. Taha, G. Subramanyam and R. E. Pino: Advances in Neuromorphic Memristor Science and Applications, Springer Series in Cognitive and Neural Systems, vol. 4, ed. R. Kozma, R. E. Pino and G. E. Pazienza (Springer, Netherlands, 2012) 211.

\section{Introduction}

Nanocrossbar arrays consist of a set of bit and word lines with a thin layer of resistive switching material in between. The nanocrossbar arrays with robust scaling ability down to $10 \mathrm{~nm}$, is widely regarded as a potential candidate for non-volatile memories [1]. However, sneak path problem limits the size of the arrays and increases their power consumption. To solve the sneak path problem, various solutions were proposed, such as $\mathrm{P}-\mathrm{N}$ junction type diode [2], Schottky type diode [3], complementary resistive switches (CRS) [4]. Among these proposed solutions, CRS is suitable for reducing parasitic current and making the application of large passive crossbar arrays feasible $[4,5]$. Memristors offer logic functions along with memory [6]. A CRS cell, which consists of two antiserial bipolar resistive switches (BRS), is suited IMP logic operations for high-density integration and can realize NOR and AND logic functions with two CRS cells which are connected via a wired AND ('\&') in two sequential cycles [7]. A single CRS cell can realize 14 Boolean functions in at most three sequential cycles [8]. These approaches are cost-effective and then improve the logic functions of CRS devices.

Fig. 1 shows the finite state machine (FSM) of the CRS cell in [7]. In Fig. 1 (a), two metal nanowires $T_{1}$ and $T_{2}$ are in orthogonal and with a CRS cell on their junction. In Fig. 2 (b), the FSM has two states, "0" and "1". Two inputs $T_{1}$ and $T_{2}$ trigger state transitions. There are two actions will change the states. State "1" changes to " 0 " when $T_{1}=$ "1" and $T_{2}=$ " 0 ". Whereas state " 0 " changes to " 1 " when $T_{1}=$ " 0 " and $T_{2}=$ " 1 ". If state $S$ changes, a current spike occurs. Thus, output $M=$ "0". Otherwise, output

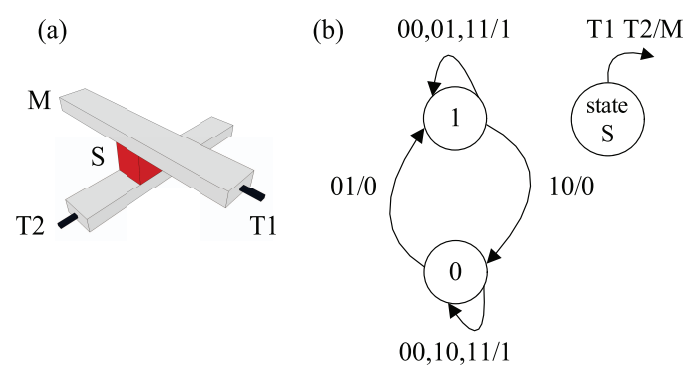


$M=$ "1". For a crossbar unit with a single CRS cell, when two voltage variables are applied to $T_{1}, T_{2}$ respectively, the next state $S^{+}$and its present state $S$, the output $M$ and inputs $T_{1}, T_{2}$, and present state $S$ are described by (1), where $T_{1}{ }^{\prime}$ is the complement of $T_{1}$.

$$
\left\{\begin{array}{l}
S^{+}=S T_{2}+T_{1}^{\prime} T_{2}+S T_{1}^{\prime} \\
M=T_{2}^{\prime} S^{\prime}+T_{1} T_{2}+T_{1}^{\prime} S
\end{array}\right.
$$

\section{Designs and simulations}

Based on the FSM of the crossbar unit with a single CRS cell shown in Fig. 1, the crossbar unit with two CRS cells that are connected via a wired AND ('\&') can be described as an FSM (Fig. 2). In Fig. 2 (a), from top to bottom, three metal nanowires are labeled by $T_{1}, T_{3}, T_{2}$ respectively, configuration bits are labeled by $S_{1}, S_{2}$. The metal nanowire $T_{3}$ is employed as a potential input and also as a current output, which is labeled by $M$. Fig. 2 (b) shows an FSM of the crossbar unit. There are four states of $S_{1}$ and $S_{2}$ including "00", "01", "10", "11". When an input vector " $110 "\left(T_{1}=\right.$ "1", $T_{2}=$ "1", $T_{3}=$ "0") is applied, all states will change to "01" $\left(S_{1}=\right.$ "0", $S_{2}=$ "1"). All states will change to states "10" $\left(S_{1}=\right.$ "1", $S_{2}=$ "0") when the input vector is "001" $\left(T_{1}=\right.$ "0", $T_{2}=$ "0", $T_{3}=$ "1"). Moreover, states will change from "01" to "00", "11", when input vectors are "101", "011" respectively. States will change from "10" to "00", " $11 "$, when input vectors are "100", "010" respectively. Either $S_{1}$ or $S_{2}$ changes, a current spike can be detected. Thus, $M=$ "0". Otherwise $M=$ "1". When three voltage variables are applied to $T_{1}, T_{2}, T_{3}$ respectively, the next states, and its present states $S_{1}, S_{2}$ are described by (2). The output $M$ and inputs $T_{1}, T_{2}, T_{3}$, and present states $S_{1}, S_{2}$ is described by (3).

$$
\begin{gathered}
\left\{\begin{array}{c}
S_{1}{ }^{+}=S_{1} T_{3}+T_{1}{ }^{\prime} T_{3}+S_{1} T_{1}{ }^{\prime} \\
S_{2}{ }^{+}=S_{2} T_{2}+T_{3}{ }^{\prime} T_{2}+S_{2} T_{3}{ }^{\prime}
\end{array}\right. \\
M=T_{1}{ }^{\prime} T_{2}{ }^{\prime} T_{3}{ }^{\prime}+S_{1}{ }^{\prime} S_{2} T_{3}{ }^{\prime}+T_{1}{ }^{\prime} S_{2} T_{3}{ }^{\prime}+ \\
+T_{1} T_{2} T_{3}+S_{1} S_{2}{ }^{\prime} T_{3}+S_{1} T_{2} T_{3}+S_{1}{ }^{\prime} S_{2}{ }^{\prime} T_{1} T_{2}{ }^{\prime}
\end{gathered}
$$

A one-bit comparator consisting of three logics among its output and two inputs $p, q$ is described by (4). One-bit half adder adds two one-bit numbers

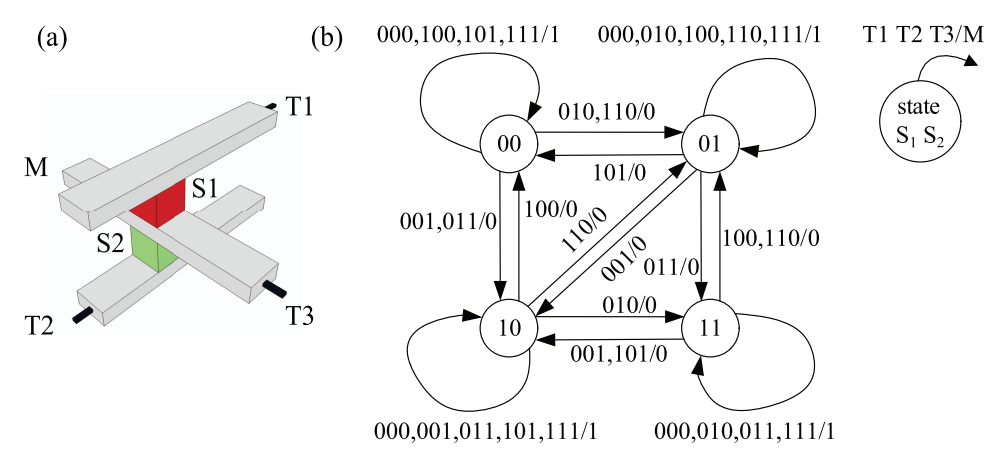

Fig. 2. (a) Crossbar unit with two CRS cells which are connected via a wired AND ('\&'). (b) FSM. 
and produces a carry bit $(\mathrm{C})$ and sum bit $(\mathrm{Su})$ as the output, its functions is described by (5).

$$
\begin{aligned}
& \left\{\begin{array}{l}
F_{p<q}=p^{\prime} q \\
F_{p>q}=p q^{\prime} \\
F_{p=q}=p^{\prime} q^{\prime}+p q
\end{array}\right. \\
& \left\{\begin{array}{l}
C=p q \\
S u=p^{\prime} q+p q^{\prime}
\end{array}\right.
\end{aligned}
$$

Fig. 3 shows the design of one-bit comparator with a single CRS cell in 7 sequential cycles. Three functions of the comparator will be realized by the same device repeatedly. Logic inputs are applied in sequence. $M=\left(p^{\prime} q\right)^{\prime}$ in the second cycle, which derives from initializing state $S$ to " 0 " in the previous cycle. $M=\left(p q^{\prime}\right)^{\prime}$ in the fourth cycle, which derives from initializing $S$ to "1" in the third cycle. $M=p q+p^{\prime} q^{\prime}$ in the seventh cycle, which derives from initializing $S$ to $p$ in the sixth cycle, and initializing $S$ to " 0 " in the fifth cycle. The outputs in the second, fourth, seventh cycles are detected whether $p$ is great than, less than or equal to $q$, respectively. If a current spike occurs in the second or fourth cycle, $p<q$ or $p>q$. Otherwise, if there is no current spike in the seventh cycle, $p=q$.

Fig. 4 shows two outputs of one-bit half adder are realized in the same device repeatedly in 5 sequential cycles. $M=p q$ in the second cycle, which derives from initializing the top configuration bit $S_{1}$ to "0", the bottom configuration bit $S_{2}$ to "1" in the previous cycle. $M=p^{\prime} q+p q$ ' in the fifth cycle, which derives from initializing $S_{1}$ to " 0 " in the third cycle, $S_{2}=$ "0" in the fourth cycle. The current spikes in the second and fifth cycle are monitored to ascertain the carry bit and sum bit of $p+q$.

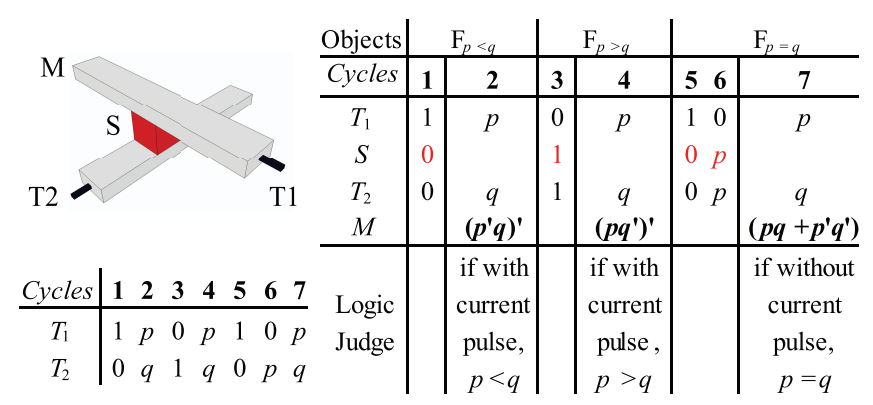

Fig. 3. One-bit comparator design. Three functions will be realized by the same device.

Fig. 5 (a) shows the memristor simulation model described in [9]. Two terminals TE and BE that represent the top and bottom electrodes of the BRS memristor respectively. The simulation model in LTspice is introduced in [10]. In this simulation, memristor with the parameters $V_{p}=0.65 \mathrm{~V}, V_{n}$ $=0.56, A_{p}=1000000000, A_{n}=1000000000, x_{p}=0.3, x_{n}=0.5, \alpha_{p}=1, \alpha_{n}$ $=6.2, a_{1}=0.00014, a_{2}=0.00014, b=0.05, x_{0}=0.99$, and $\eta=-1$. We use the circuits (Fig. 5 (b), (c)) to simulate one-bit comparator and one-bit half adder in LTspice. In Fig. 5 (b), two antiserial BRS elements in which one BE 


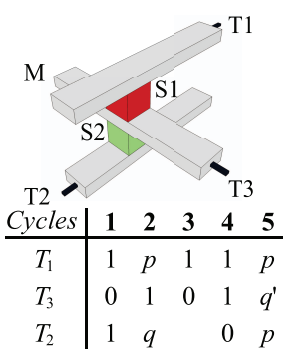

\begin{tabular}{|c|c|c|c|c|c|}
\hline Objects & \multicolumn{2}{|c|}{$C=p q$} & \multicolumn{3}{|c|}{$S u=p^{\prime} q+p q^{\prime}$} \\
\hline Cycles & 1 & 2 & 3 & 4 & 5 \\
\hline$T_{1}$ & 1 & $p$ & 1 & 1 & $p$ \\
\hline$S_{1}$ & 0 & & 0 & & \\
\hline$T_{3}$ & 0 & 1 & 0 & 1 & $q^{\prime}$ \\
\hline$S_{2}$ & 1 & & & 0 & \\
\hline$T_{2}$ & 1 & $q$ & & 0 & $p$ \\
\hline$M$ logic & & $M=p q$ & & & $M=p^{\prime} q+p q^{\prime}$ \\
\hline
\end{tabular}

Fig. 4. One-bit half adder design. Two functions are realized in the same device.

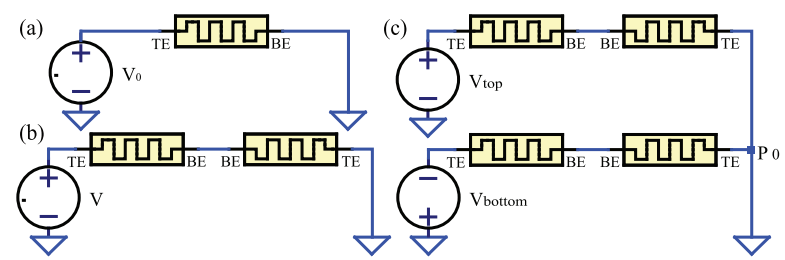

Fig. 5. Simulation circuits of the crossbar units. (a) Circuit used to carry out a BRS cell simulation. (b) Simulation circuit of a one-bit comparator. (c) Simulation circuit of a one-bit half adder.

terminal is connected to the other BE terminal directly. In Fig. 5 (c), two CRS cells intersect at $P_{0} . V_{\text {top }}$ and $V_{\text {bottom }}$ are the voltage variables applied to the top and bottom CRS cells respectively.

Fig. 6 shows the simulation results of a one-bit comparator. A voltage, whose amplitude equals to $1.4 \mathrm{~V}$ and period equals to $30 \mathrm{nsec}$, is applied. If there is a current spike in $30-60$ nsec, or in $90-120$ nsec, $p<q$ or $p>q$ respectively, whereas if there is no current spike in $180-210$ nsec, $p=q$. Fig. 7 shows the simulation results of a one-bit half adder. The amplitudes of $V_{\text {top }}$ and $V_{\text {bottom }}$ are $1.4 \mathrm{~V}$ and their periods are $30 \mathrm{nsec}$. The output logic in $30-60$ nsec is the carry bit of $p$ and $q$, whereas the output logic in 120 $150 \mathrm{nsec}$ is the sum bit of $p$ and $q$.

Synthesis results in $45 \mathrm{~nm}$ CMOS process with Synopsys Design Compiler show that the areas of one-bit comparator and the one-bit half adder are $3.724 \mu \mathrm{m}^{2}$ and $2.66 \mu \mathrm{m}^{2}$ respectively. The crossbar units, which are fabricated by stacking $10 \mathrm{~nm} \times 10 \mathrm{~nm} \mathrm{HfO}_{\mathrm{x}}$ crossbar resistor RAM elements [1], are three orders of magnitude less than the CMOS designs.

\section{Conclusions}

In this letter, we first present the FSM of the crossbar unit with two CRS cells which are connected via a wired AND ('\&'). Then, a one-bit comparator and a one-bit half adder are designed using the crossbar units with one or two CRS cells respectively. Simulations results show that the crossbar units can realize the one-bit comparator and the one-bit half adder with three orders of magnitude less than those in $45 \mathrm{~nm}$ CMOS process. The designs of one-bit comparator and one-bit half adder with CRS crossbar units can reinforce the logics of crossbar circuits and allows further gains in terms of chip area, and 

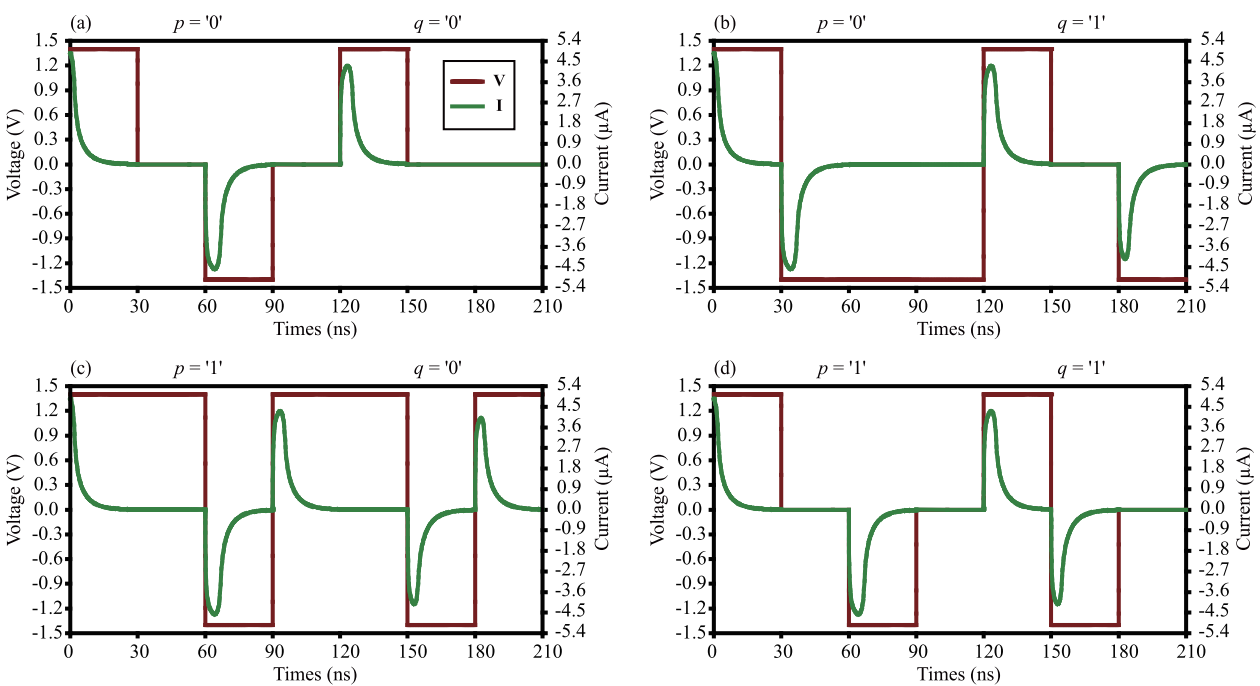

Fig. 6. Simulation results of one-bit comparator with voltage-time and current-time characteristics for all possible combinations of inputs $p$ and $q$.
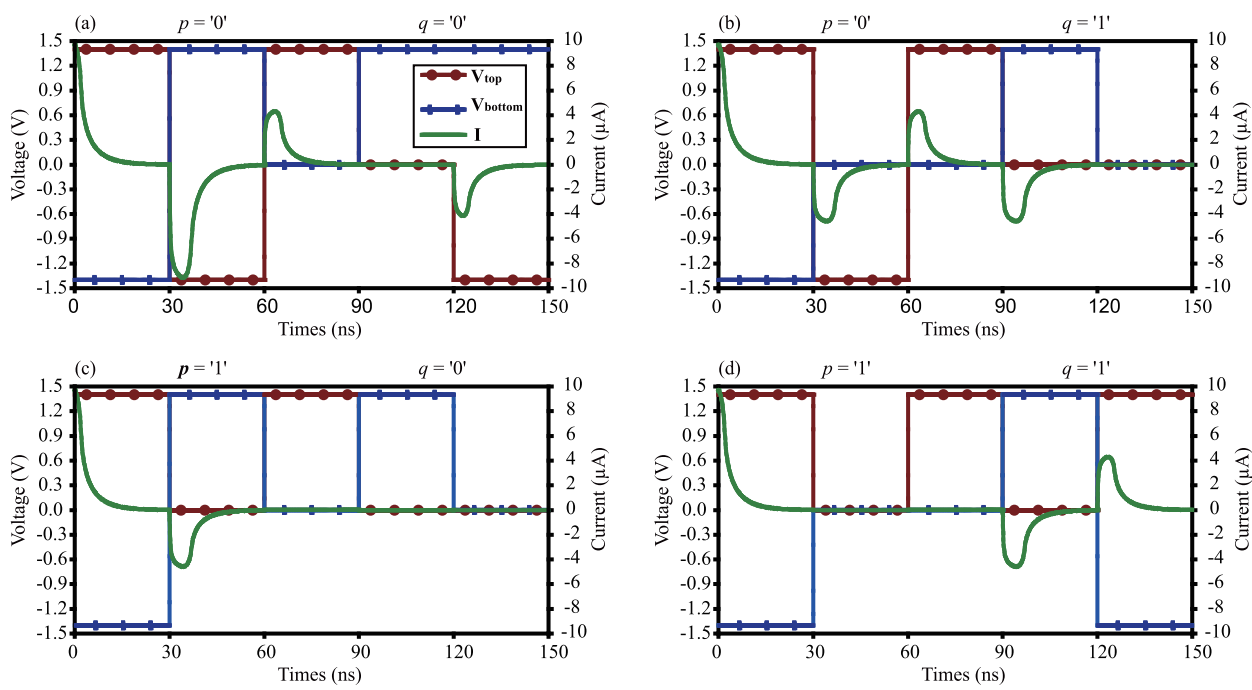

Fig. 7. Simulation results of one-bit half adder with voltage-time and current-time characteristics for all possible combinations of inputs $p$ and $q$.

integration density.

\section{Acknowledgments}

This paper is partly supported by Program for New Century Excellent Talents in University (NCET-12-0165), the National Science Foundation of China under Grant No. 61070194, and the Science \& Technology Key Projects of Hunan Province (2011FJ2003). 